\title{
Piezoceramic cantilever sensor design for weak-impact detection on plates
}

\author{
Young-Sup Lee ${ }^{\mathrm{a}, *}$, Sang-Kwon Lee ${ }^{\mathrm{b}}$ and Kihong Shin ${ }^{\mathrm{c}}$ \\ ${ }^{a}$ Department of Embedded Systems Engineering, University of Incheon, Yeonsu-Gu, Incheon, South Korea \\ ${ }^{\mathrm{b}}$ Department of Mechanical Engineering, Inha University, Nam-Gu, Incheon, South Korea \\ ${ }^{\mathrm{c}}$ Department of Mechnical and Automotive Engineering, Andong National University, Andong, Kyungpook, South \\ Korea
}

\begin{abstract}
A piezoelectric cantilever type sensor for locating the precise weak-impact or touch position on a plate is presented in this paper. Since the importance of human-computer interface such as a touch panel system has been rapidly increasing recently, this study could suggest an appropriate sensor for the detection of a weak-impact point effectively and accurately for such a system. This sensor detects the out-of-plane vibration of a panel when a touch with a finger or pen is applied on it. The sensor is made with a steel beam and a single crystal PMN-PT patch is bonded on the beam, which is designed to detect the base vibration of the panel. The sensor was designed, manufactured to verify the detect ability of a weak-impact and attached on two different plates of a glass of $400 \times 400 \times 4 \mathrm{~mm}$ and a wooden MDF of $600 \times 600 \times 9 \mathrm{~mm}$. The experiment result of the sensor was compared with that of an accelerometer which can also be used for the same purpose and shows clear weak-impact responses with a narrow-band property at its resonant frequency. It is expected that the cantilever type sensor in this study could be applied to make a simple flat plate into a touch panel when the time difference of arrivals method is used to locate the weak-impact point.
\end{abstract}

Keywords: Piezoelectric cantilever sensor, weak-impact locating, wave dispersion, narrow-band response, time difference of arrivals

\section{Introduction}

Touch panels have been essential input devices for various computer systems. Various human-computer interface technologies, such as the optical method, electrostatic capacitive method, ultrasonic method, resistive membrane method, and so on, are available commercially in order to implement touch panels [1]. By the way, the above methods require high costs although they could provide quick responses and exact touch positions on panels [1].

In this paper, a cantilever type beam with a piezoelectric patch is considered to implement a new weak-impact or touch detection sensor. The cantilever sensor can be installed on a simple flat and thin plate, such as a commercial white board in order to make it into a touch panel. Since a vibration occurs during impacting weakly or touching on such a plate with a tool like a pencil, those sensors based on detecting vibration may be used to implement a touch panel. Hence the sensor needs to effectively detect a weak-impact or touch imposed on the plate. The weak-impact in this study represents a relatively very small magnitude impact which is similar to a touch with a tool like a pen on a plate.

Since a piezoelectric transducer bonded on a vibrating structure is a strain-integrating element, its output has a relationship with the flexural motion of the structure [2]. Because of this reason, a number of researchers have studied many sensors based on piezoelectric transducers in order to apply on beams and plates. Lee and O'Sullivan

\footnotetext{
*Corresponding author: Young-Sup, Lee, Department of Embedded Systems Engineering, University of Incheon, 12-1 Songdo-Dong, Yeonsu-Gu, Incheon, South Korea. E-mail: YSL@incheon.ac.kr.
} 
suggested a piezoelectric strain rate gauges by arranging piezoelectric rectangular patches [3]. Distributed piezo sensors covering a large area of a structure have been also investigated because their spatial shaping enables piezo sensors to measure specific physical quantities without complex post-processing [4-6]. Some of these are the uniformly distributed sensor for gradient sensing [4], quadratically shaped sensor for volume velocity sensing [5], and triangularly shaped sensor for tip position sensing [6]. These examples are coupled sensors responding to all the structural modes of a system, which can be distinguished from the modal sensors, in which the modal sensors respond only to a specific vibration mode [4]. Cantilever type piezo sensors are also studied theoretically and experimentally for various purposes, for example the sensor effect of piezoelectric benders [7], chemical and biomedical detection [8] and the sensing effects of piezoelectric multimorph cantilevers [9].

Basically accelerometers for measuring plate vibration, microphones for detecting impact sound, or ultrasonic sensors for catching air-transmitted ultrasound are commercially available sensors to implement a locating sensor system for a touch panel. Although these sensors are different from each other, they can detect a weak-impact or touch on a plate [10].

The cantilever sensor in this study consists of a small steel cantilever beam and a piezoelectric patch which is bonded on the beam. The sensor will be attached on a flat and thin plate. When the plate vibrates due to a weak-impact or touch imposed on it, the sensor will detect the vibration as a base vibration if the sensor is attached on the plate firmly enough $[10,11]$. Then the piezo patch on the sensor will generate an electric charge output which is proportional to the plate vibration as well as beam vibration [10-13]. Thus the cantilever piezoelectric sensor can be used as an alternative sensor to detect a weak-impact or touch. A locating sensor system using an array of those sensors can be applied for implementing a new touch panel effectively when the time difference of arrivals (TDOA) approach is applied $[14,15]$. It is well-known that the TDOA approach has been used in many applications such as SONAR and RADAR for location identification of objects. So this cantilever sensor concept in this paper is a fundamental study to implement a touch locating sensor for transforming a commercial thin plate into a touch panel.

Background for the cantilever sensor concept is investigated in Section 2. The sensor design is described in Section 3 and the response of the sensor is also discussed theoretically. In Section 4, manufacture, experimental results, and analysis are presented with actual measured data. Finally conclusions of this study are written in Section 5 .

\section{Concept of cantilever sensor approach}

As illustrated in Fig. 1, a vibration wave is generated by a touch or weak-impact on a plate where three relevant sensors are installed on the plate at predefined locations with $D$ and $d$. For this purpose, a commercial accelerometer could be one of good sensors because it is a representative sensor for vibration detection and gives a broadband property below its fundamental natural frequency [10]. The touch point $(x, y)$ can be calculated if the vibration wave propagation velocity and the time difference of the wave arrivals at the three sensors are known [14,15]. Some references suggest specific equations to estimate the weak-impact point precisely, in which the flexural wave propagation velocity $c$ of a plate and the arrival time differences $\Delta t_{12}=t_{1}-t_{2}, \Delta t_{13}=t_{1}-t_{3}$, and $\Delta t_{23}=t_{2}-t_{3}$ are critical factors for precise pointing [14,15]. The time differences $\Delta t_{12}, \Delta t_{13}$, and $\Delta t_{23}$ are usually calculated with the cross-correlation function.

If a piezo patch is directly bonded on a vibrating thin plate due to a weak-impact or touch, the piezo patch will detect various vibration waves, such as the in-plane longitudinal, out-of-plane flexural, and transverse shear waves $[16,17]$. It indicates that the electric signal from the directly-bonded piezo patch contains the dispersive wave motions like the out-of-plane flexural motion, in which the wave velocity on a plate varies with frequency [13] as expressed with the following equation for the phase velocity of the flexural motion against frequency $\omega[16]$.

$$
c_{b}=\omega^{1 / 2}(D / m)^{1 / 4}
$$

where $m$ is the mass per unit area of the plate and the bending stiffness, $D=E h_{p}^{3} / 12\left(1-v^{2}\right)$ in which $E, h_{p}$ and $v$ are Young's modulus, the plate thickness and the Poisson's ratio respectively. Thus, since it is known that a piezo 


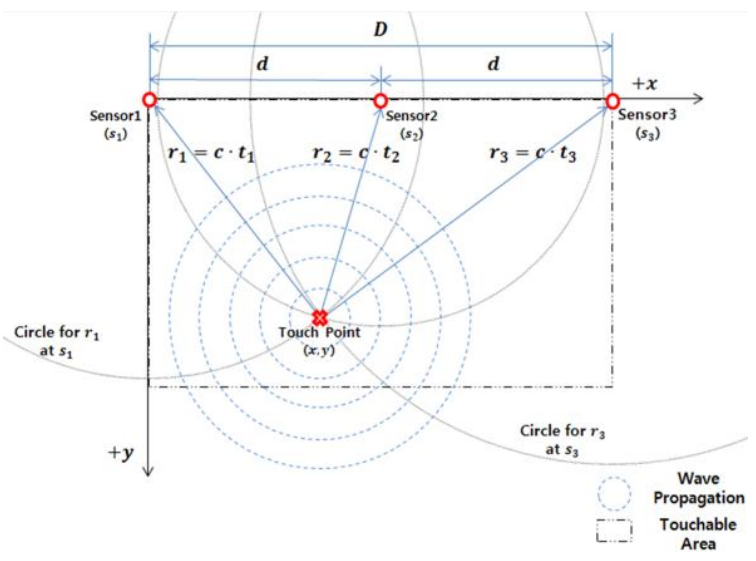

Fig. 1. Principle of locating a touch point using the TDOA approach with sensors on a plate.

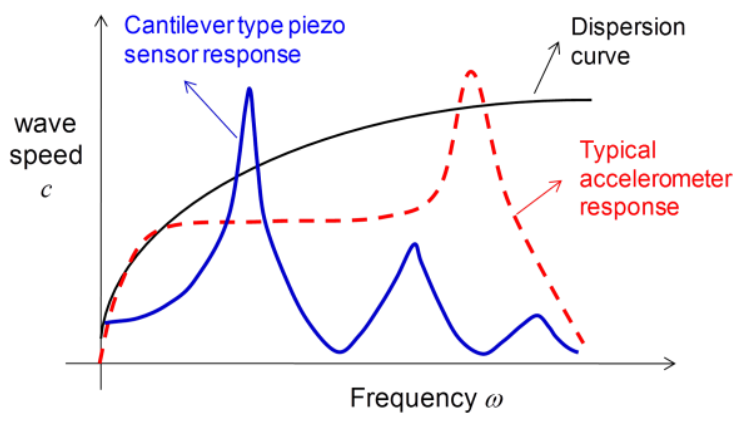

Fig. 2. Required narrow-band property for the cantilever type sensor.

patch can detect vibration waves in a very wide frequency range [13], the dispersive component in the piezo output signal makes the determination of the constant wave velocity, which is critically important to locate the touch position by the TDOA method, very difficult.

Because of the above reason, the cantilever beam sensor concept is considered in this study. In order to offer a constant wave velocity, the frequency response of the sensor is necessary to have a narrow band property as illustrated in Fig. 2. By the way, the accelerometer gives a broadband property below its fundamental natural frequency [12] as plotted in Fig. 2. The electric signal from the piezo patch bonded on a cantilever beam provides the narrow band property at the resonance frequencies of the cantilever beam. The plate, in which the cantilever type sensor is installed, will be exposed to an inertial force when it experiences the out-of-plane flexural motion by a weak-impact or touch. Then the cantilever on the plate will vibrate out-of-plane especially at the resonant frequencies of the beam itself. As the cantilever has the largest bending vibration motion at its fundamental natural frequency, the piezo patch, which is bonded on the cantilever, can generate the electrical signal corresponding to the cantilever motion.

The frequency response of the cantilever beam installed on the plate works as a mechanical filter to pass only the flexural wave very sensitively, which is the transmitted one among the various waves from the vibrating plate. The piezo patch on the beam generates the corresponding electric signal while the cantilever type sensor can achieve the narrow band property at the first resonant mode of the cantilever beam and allows offering a constant bending wave velocity for the TDOA method.

\section{Cantilever type sensor design}

\subsection{Fundamental natural frequencies of cantilever sensor}

As shown in Fig. 3, a cantilever type sensor which is made with a steel cantilever beam (length $\times$ width $\times$ thickness $\left.=L_{b} \times B \times t_{b}\right)$ and a piezoelectric transducer $\left(L_{p} \times B \times t_{p}\right)$ is attached on a plate. If a weak-impact by a pen is applied at a point on the plate, the plate vibration will be transmitted as a base vibration to the cantilever sensor.

The fundamental natural frequency of the cantilever type sensor can be calculated as the following [18]:

$$
f_{n}=\frac{k_{n}^{2}}{2 \pi L_{b}^{4}} \sqrt{\frac{E_{b} I_{e}}{m_{b}}}
$$

where $L_{b}$ is the beam length, $m_{b}$ is the mass per unit length of the beam, $E_{b}$ is Young's modulus, $I_{e}$ is the second moment of the beam section and $k_{n}$ are $1.8751,4.6941,7.8548,10.9955$ and 14.1372 when $n=1$ to 5 respectively, 


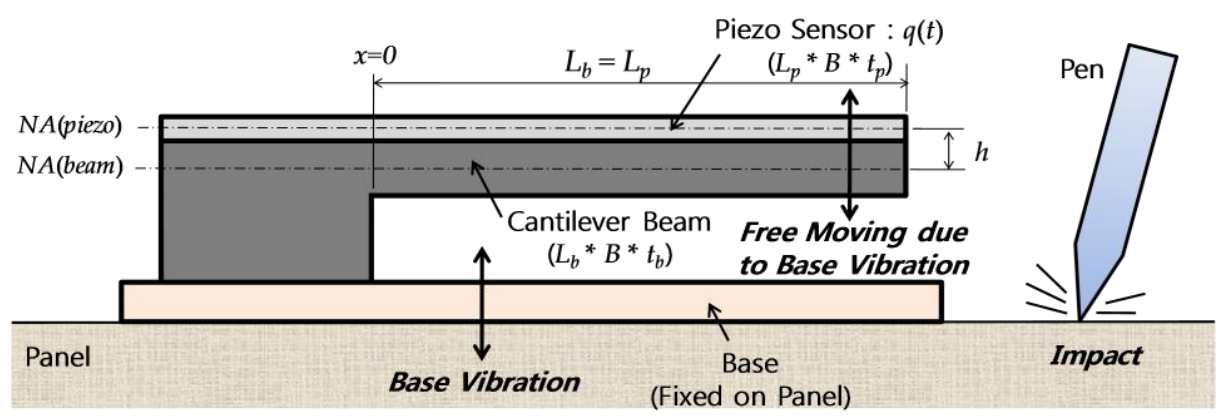

Fig. 3. Design of a cantilever beam sensor with a piezoelectric transducer.

and $(2 n-1) * \pi / 2$ if $n$ is greater than 5 . Figure 4 shows the estimated fundamental natural frequencies of the cantilever sensor when the thickness of the piezo transducer is $t_{p}=0.3 \mathrm{~mm}$ but the thickness and the length of the steel beam are varied as $t_{b}=0.1-1.0 \mathrm{~mm}$ and $L_{b}=10.0-20.0 \mathrm{~mm}$ respectively. Since the Euler-Bernoulli beam model was used, the variation of the beam width was not considered. As shown in Fig. 4, the fundamental natural frequencies can be chosen between $f_{1}=500-9,000 \mathrm{~Hz}$ with the various dimensions.

\subsection{Response of cantilever sensor}

It is well-known that a piezo transducer can generate an electrical charge output proportional to the strain of the piezo itself [13]. The strain of the piezo patch, which is bonded on the cantilever beam when pure flexural motion is applied, has a relationship with the beam flexural displacement [6]. Thus the electric charge output of the piezo patch can be expressed as [6,13]

$$
q(t)=e_{31} h B \int_{0}^{L_{b}} S(x) \frac{d^{2} w}{d x^{2}} d x
$$

where $e_{31}$ is a piezoelectric constant, $B$ is the beam width which is equivalent to the piezo width, the distance between the neutral axes of the beam and the piezo patch $h=\left(t_{b}+t_{p}\right) / 2$, and $S(x)$ is a shape function of the piezo. In this case $S(x)=1$, because the piezo patch shape is exactly equal to that of the beam.

The flexural out-of-plane displacement of the cantilever beam $w$ can be defined using the modal summation method as [18]

$$
w=\sum_{n=1}^{\infty} A_{n}(t) \phi_{n}(x)
$$

where $A_{n}(t)$ is the modal amplitude and $\phi_{n}(x)$ is the mode shape function. After some manipulation, Eq. (4) can be expressed, by using $S(x)=1$ as the following [6]:

$$
q(t)=e_{31} h B \sum_{n=1}^{\infty} A_{n}(t) \int_{0}^{L_{b}} \frac{d^{2} \phi_{n}(x)}{d x^{2}} d x
$$

If the flexural out-of-plane displacement of the plate, where the cantilever sensor is attached on, is symbolized as $z(t)$, then the inertial force exerted to the cantilever beam due to the plate vibration is $-m(x) \ddot{z}(t)$, where $m(x)$ is the beam mass per unit length [11]. The (-) sign indicates that the beam flexural motion $w(t)$ is out-of-phase with the plate flexural motion $z(t)$. Thus the modal amplitude $A_{n}(t)$ of the cantilever beam caused by the inertial force can be expressed with 


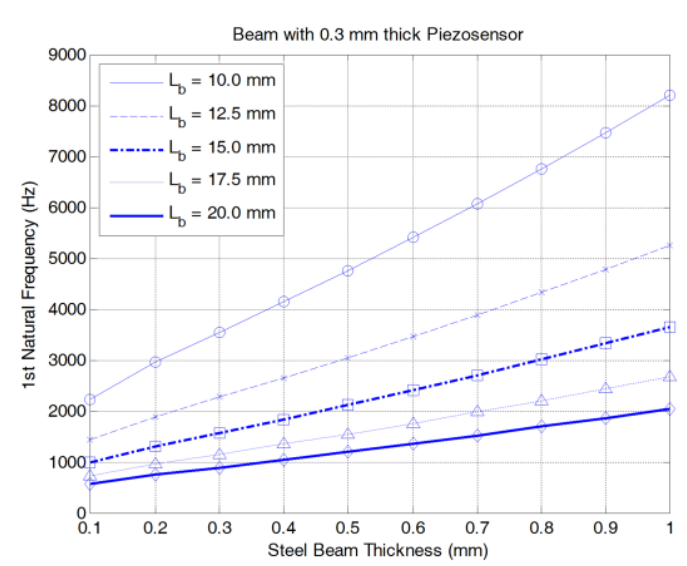

Fig. 4. Estimation of fundamental natural frequencies of a cantilever beam with a piezo transducer.
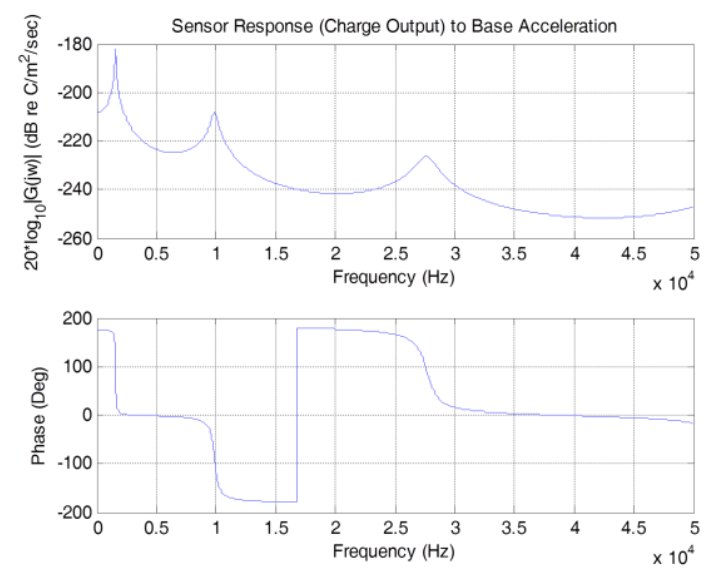

Fig. 5. Frequency response function of a cantilever sensor below $50,000 \mathrm{~Hz}$.

$$
A_{n}(t)=\sum_{n=1}^{\infty} \frac{-\int_{0}^{L_{b}} m(x) \ddot{z}(t) \phi_{n}(x) d x}{M_{n}\left[\left(\omega_{n}^{2}-\omega^{2}\right)+j 2 \zeta_{n} \omega_{n} \omega\right]}
$$

where $\zeta_{n}$ is the damping ratio and $m(x)=m_{o}=\rho A$ is the mass per unit length of the beam. Thus the frequency response of the sensor charge output against the inertial motion (acceleration) of the plate base vibration can be defined by the following:

$$
G(j \omega)=\frac{q(t)}{\dddot{z}(t)}=-e_{31} h B \sum_{n=1}^{\infty} \frac{m_{o}\left[\phi_{n}^{\prime}\left(L_{b}\right)-\phi_{n}^{\prime}(0)\right] \int_{0}^{L_{b}} \phi_{n}(x) d x}{M_{n}\left[\left(\omega_{n}^{2}-\omega^{2}\right)+j 2 \zeta_{n} \omega_{n} \omega\right]}
$$

Thus the theoretical frequency response function of the output signal from the cantilever sensor against the plate base vibration can be plotted as shown in Fig. 5, using the above equations when $L_{b} \times B \times t_{b}=15 \times 14 \times 0.3 \mathrm{~mm}$ and $L_{p}$ $\times B \times t_{p}=15 \times 14 \times 0.3 \mathrm{~mm}$. The physical dimensions and mechanical properties of the cantilever beam and the piezo transducer are summarized in Table 1 .

Table 1

Dimensions and properties of the cantilever beam the piezo transducer

\begin{tabular}{ccc}
\hline & Dimension & Remarks \\
\hline Beam & $15 \times 14 \times 0.3 \mathrm{~mm}$ & Steel $L_{b} \times B \times t_{b}$ \\
Piezosensor & $15 \times 14 \times 0.3 \mathrm{~mm}$ & $L_{p} \times B \times t_{p}$ \\
$\zeta_{n}$ & 0.05 & Damping ratio $(5 \%)$ \\
$h=\left(t_{p}+t_{b}\right) / 2$ & $0.3 \mathrm{~mm}$ & Distance between neutral axes of beam and piezosensor \\
$m_{o}$ & 0.0647 & Mass per unit length \\
$M_{n}$ & $9.7020 * 10^{-4}$ & Modal mass \\
\hline
\end{tabular}

Figure 5 shows the frequency response function of the designed cantilever sensor as the 1st, 2nd, and 3rd natural frequencies are about $1,500 \mathrm{~Hz}, 10,000 \mathrm{~Hz}$, and $27,500 \mathrm{~Hz}$ respectively. The 1 st natural frequency of the cantilever sensor at $1,600 \mathrm{~Hz}$ is the same frequency as plotted in Fig. 4. The cantilever sensor is designed to have a highly sensitive response only around the 1 st resonant frequency and this response will give a narrowband property to offer a constant bending wave velocity. 
As shown in Fig. 5, the phase response below the fundamental natural frequency is 180 degrees different (out-of-phase) against the base vibration input. This indicates the cantilever sensor oscillates to the opposite direction against the base vibration input below the 1st resonant frequency of the beam. But the phase response between the 1st and 2nd natural frequencies is in-phase. It is noted that a phase drop of 180 degrees occurs continuously at every resonance since the cantilever sensor motion is governed by the base vibration.

\section{Manufacturing and weak-impact test results}

\subsection{Manufacturing of cantilever sensor}

A cantilever type sensor with a piezoelectric transducer was designed in section 3 and then it was manufactured as shown in Fig. 6. The sensor was made with a rectangular cantilever beam (steel, $L_{b} \times B \times t_{b}=15 \times 14 \times 0.3 \mathrm{~mm}$ ), a circular foundation (steel, $\phi 25 \times 0.3 \mathrm{~mm}$ thick), a support (steel, $25 \times 5 \times 1 \mathrm{~mm}$ thick) and a piezo transducer. Especially the piezo transducer is a single crystal PMN-PT (piezoelectric magnesium niobate - lead titanate, made by Ibule Photonics Co.) rectangular patch $\left(L_{p} \times B \times t_{p}=15 \times 14 \times 0.3 \mathrm{~mm}\right)$, and it was bonded on the beam as illustrated in Fig. 6. Generally the PMN-PT element has higher piezoelectric constants and more expensive than a piezoceramic PZT (piezoelectric zirconate titanate). The support was designed to maintain the interval of $1 \mathrm{~mm}$ between the beam and the foundation.

The output of these piezo sensor will just be used for calculating the cross-correlation function to locate the touch point on the plate. So a precise calibration of the sensor does not need for this application.

\subsection{Weak-impact test set-up}

The manufactured cantilever sensor was attached on a glass plate $(400 \times 400 \times 4 \mathrm{~mm})$ and an MDF plate $(600 \times$ $600 \times 9 \mathrm{~mm}$ ) respectively as shown in Fig. 7. The dimension and mechanical properties of the two plates are

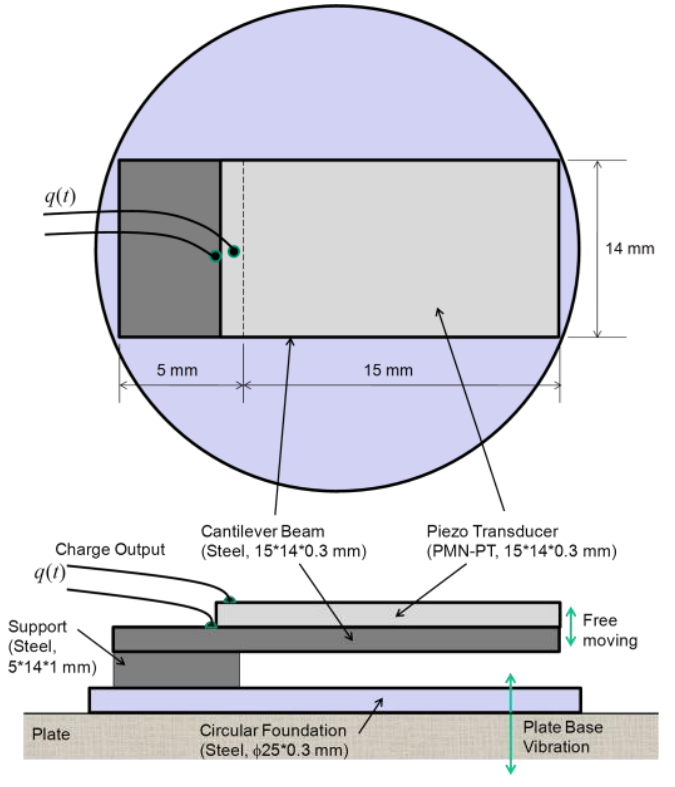

(a)
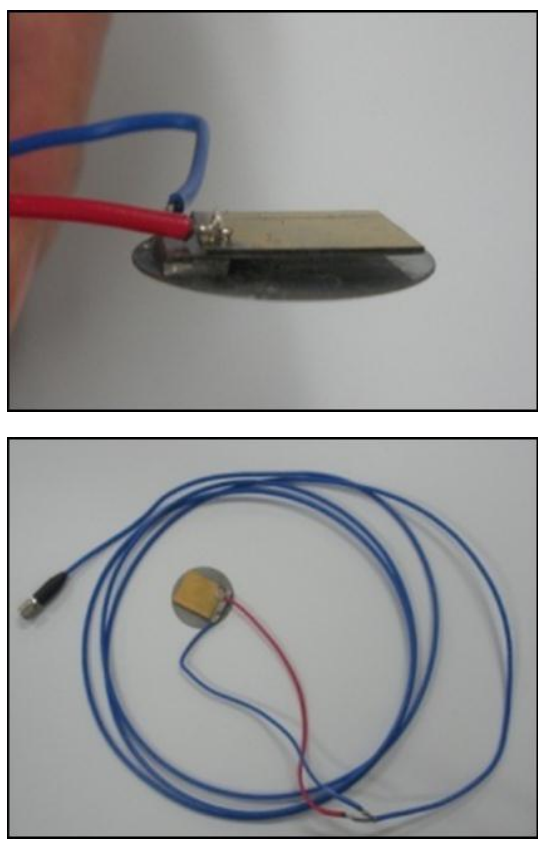

(b)

Fig. 6. A cantilever type sensor for a weak-impact or touch locating: (a) Schematic of the cantilever sensor with a PMN-PT transducer; (b) Manufactured cantilever sensor. 

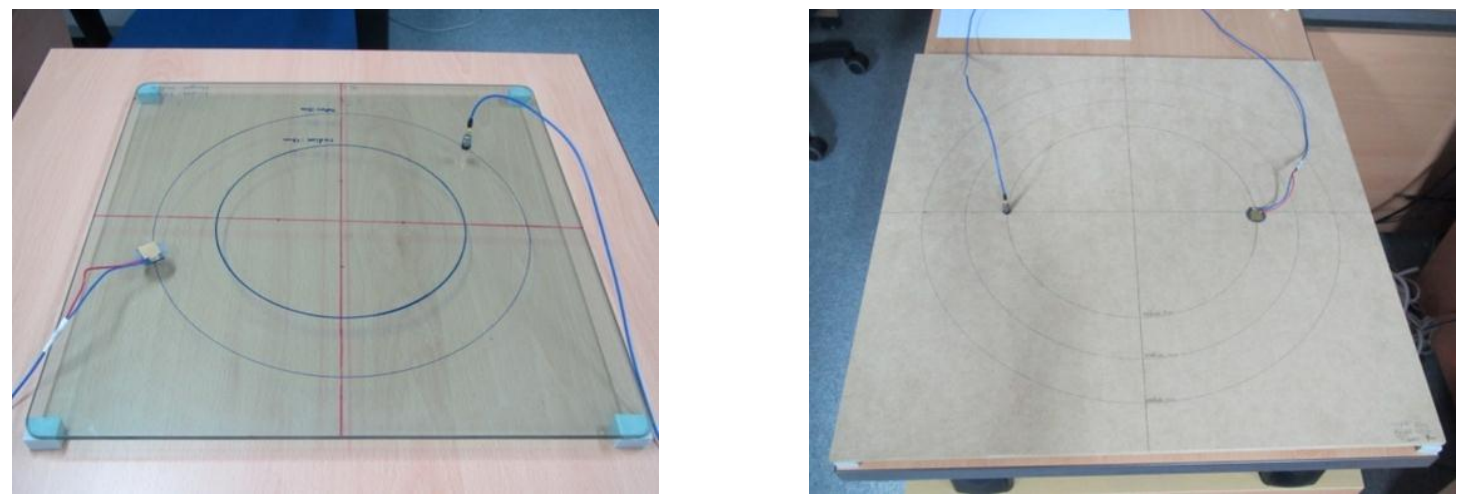

Fig. 7. Weak-impact response tests on a glass plate (Left) and an MDF plate (Right).

presented in Table 2. A PCB Piezotronics 352C66 accelerometer (resonance frequency $=40 \mathrm{kHz}$ ) was attached together with the cantilever sensor to compare their weak-impact responses. In contrast, cantilever sensor detects the beam vibration which is the mechanically filtered plate base vibration. Due to the weak-impact on the plate itself, the accelerometer detects just the plate vibration.

Table 2

Properties of the glass and the MDF plates

\begin{tabular}{ccc}
\hline & Glass plate & MDF plate \\
\hline Dimension $L_{x} \times L_{y} \times h_{p}$ & $400 \times 400 \times 4 \mathrm{~mm}$ & $600 \times 600 \times 9 \mathrm{~mm}$ \\
Density $\rho$ & $2.4 \times 10^{3} \mathrm{~kg} / \mathrm{m}^{3}$ & $6.5 \times 10^{2} \mathrm{~kg} / \mathrm{m}^{3}$ \\
Young's modulus $E$ & $6.0 \times 10^{10} \mathrm{~N} / \mathrm{m}^{2}$ & $4.6 \times 10^{9} \mathrm{~N} / \mathrm{m}^{2}$ \\
Poisson's ratio $v$ & 0.24 & 0.30 \\
Mass per unit area $m=\rho h_{p}$ & $9.60 \mathrm{~kg} / \mathrm{m}^{2}$ & $5.85 \mathrm{~kg} / \mathrm{m}^{2}$ \\
Bending stiffness per & & \\
Unit width & $339.56 \mathrm{Nm}$ & $307.08 \mathrm{Nm}$ \\
$D=E h_{p}^{3} / 12\left(1-v^{2}\right)$ & & \\
\hline
\end{tabular}

Each plate was supported by four rubbers at every corner in order to isolate the plates from ambient vibration or shocks. Some circles were drawn on both plates as shown in Fig. 7 and the two sensors (cantilever sensor and accelerometer) were positioned on the circle outline with the radius of $R=15 \mathrm{~cm}$. Weak-impacts were imposed on the center of the circles ( $\mathrm{P} 1$ point), $7 \mathrm{~cm}$ to the cantilever sensor from the center ( $\mathrm{P} 2$ point), and $5 \mathrm{~cm}$ to the accelerometer from the center (P3 point) respectively as illustrated in Fig. 8. In the test, the weak-impact was defined as a drop of a mechanical pencil with its sharp end at $3 \mathrm{~cm}$ height.

As shown in Fig. 8, the output signal from the cantilever sensor was measured by an Endevco charge amplifier model 133, an NI 9223 board, and an NI cRio-9004. In the charge amplifier, a bandpass filter was used with the lower and higher cutoff frequencies of $10 \mathrm{~Hz}$ and $10 \mathrm{kHz}$ respectively. The accelerometer signal was amplified through a 442B104 PCB signal conditioner. The three sensor signals were then post-processed at a PC. The sampling frequency of the experiment was set to $50,000 \mathrm{~Hz}$.

\subsection{Weak-impact test results and discussions}

Weak-impact response tests for the cantilever sensor and the accelerometer on the two different plates have been accomplished one by one. Figure 9 for the glass plate and Fig. 10 for the MDF plate show normalized measured responses for the sensors when a weak-impact is applied on the center of the circle (P1). 


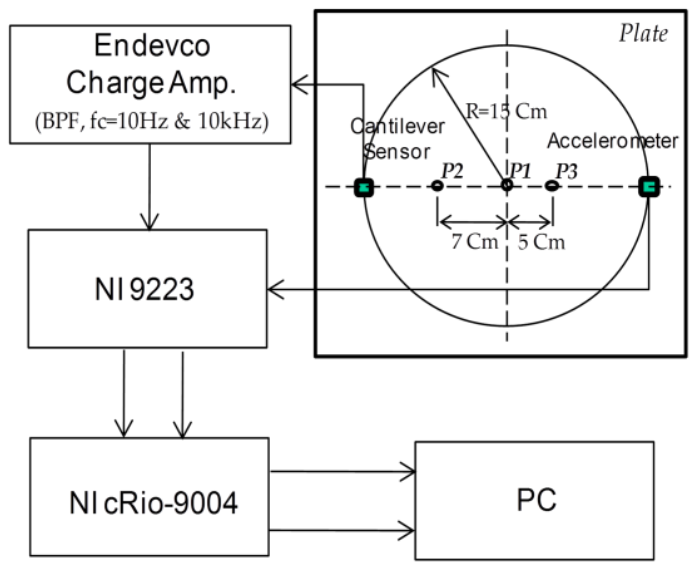

Fig. 8. Measurement set-up for tests of the sensor.

Figures $9 \mathrm{a}$ and $9 \mathrm{~b}$ show the time and the time-frequency responses of the accelerometer and the cantilever sensor respectively on the glass plate, which were measured just after the weak-impact. However, the two time responses look quite different from each other in terms of their signal wave forms and ringing durations. The reflected waves are observed from the two time signals because of the limited size of the glass plate. The signal from the cantilever sensor in Fig. 9b looks that a strong tonal component is involved. However, the signal from the accelerometer shows a broadband property as can be seen from Fig. 9a.

Also it can be explained with the normalized power spectral density (PSD) functions, which are plotted in Fig. 9c, below $10,000 \mathrm{~Hz}$ of the measured sensor responses on the glass plate. For the cantilever sensor, only one resonance at about $1,300 \mathrm{~Hz}$ is observed and this is the tonal component which is shown in the time response as a ringing signal. However, the two largest resonant modes of the accelerometer response are measured at about $1,300 \mathrm{~Hz}$ and $2,300 \mathrm{~Hz}$ respectively, which are specific vibration modes of the glass plate, and generally the PSD magnitude of the accelerometer response is larger than that of the cantilever sensor response over the frequency range between $0-10,000 \mathrm{~Hz}$ because the accelerometer is a broadband type below its fundamental natural frequency at $40 \mathrm{kHz}$ but the cantilever sensor is a narrowband one. Thus the signal energy of the cantilever sensor is concentrated mostly on the largest resonant mode, and this explains why the time response has a strong tonal component. The time-frequency response in Fig. $9 \mathrm{~b}$ explains this fact too.

Also it is known the quality factor $Q$ shows the sharpness of a resonance peak and is a good estimator to predict the decay rate in time responses. So the higher $Q$ gives a narrower band property, which is an important element for the cantilever sensor to locate impact points more precisely. It can be defined as [11]

$$
Q=\frac{f_{o}}{f_{2}-f_{1}}
$$

where $f_{o}, f_{1}$, and $f_{2}$ are the resonance frequency, the lower half-power frequency and the upper half-power frequency of the first resonant frequency. From Fig. 9c, the $Q$ factor at the resonance from the PSD of the cantilever sensor on the glass plate is $Q=1.233$

For the MDF plate, the weak-impact responses of the two sensors are plotted in Fig. 10. Since the MDF plate is less rigid and its dimension is larger than the glass plate, the time and time-frequency responses in Figs 10a and 10b respectively show that a strong tonal component is clearly observed by both sensors. Also the time responses show that the bending wave velocity at the MDF plate is lowered and this makes a reduced number of reflected waves in the same time span than the glass plate.

The PSDs of the both sensor responses in Fig. 10c demonstrate the only eminent resonance is measured at the low frequency of about $1,000 \mathrm{~Hz}$. The PSD of the accelerometer response is dramatically changed compared to the 

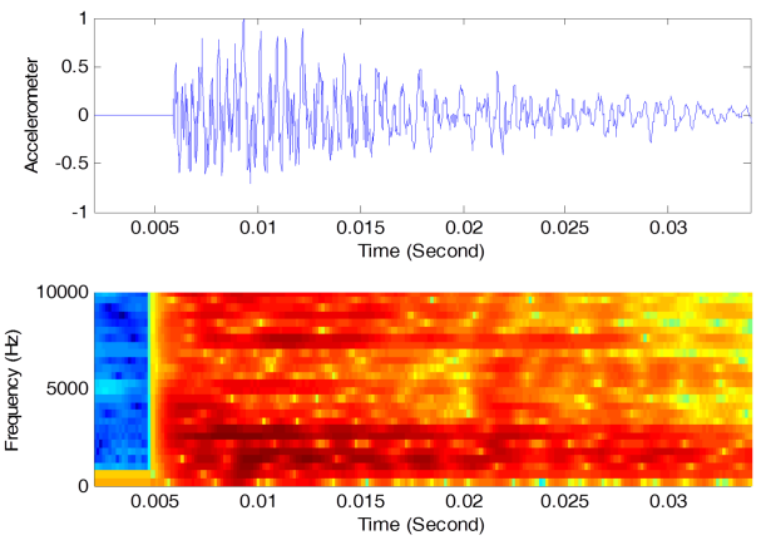

(a)
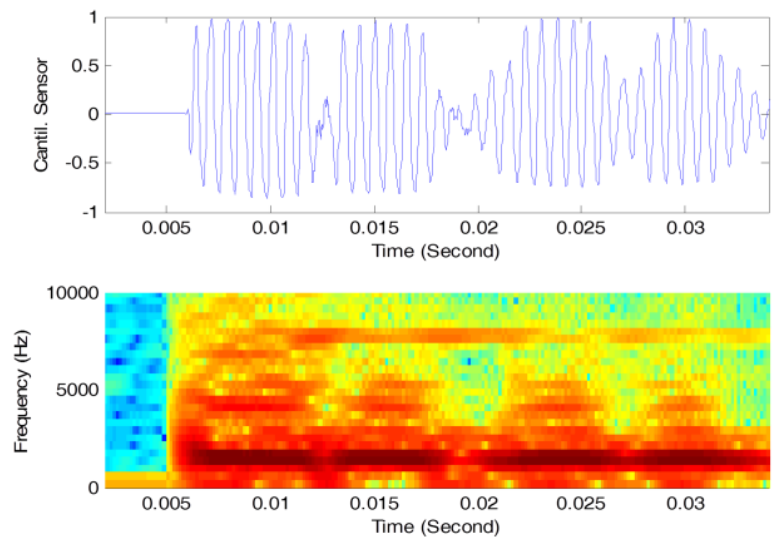

(b)

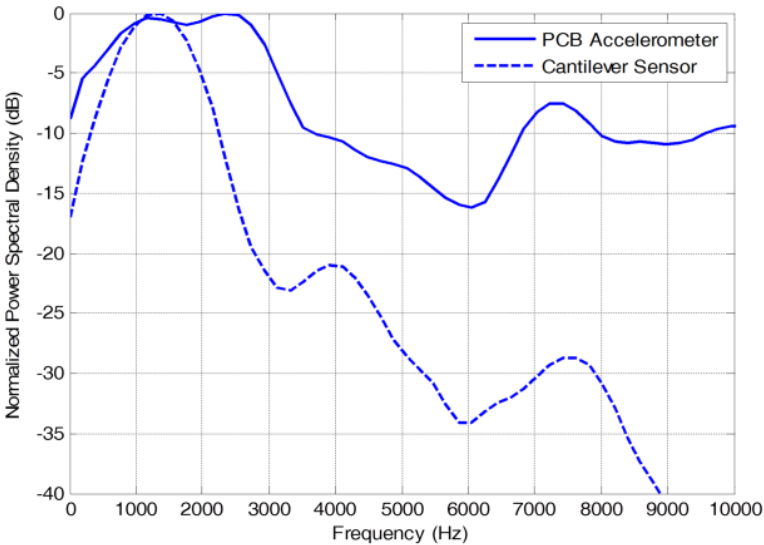

(c)

Fig. 9. Measured weak-impact responses of the two sensors on the glass plate. (a) Time and time-frequency responses of the accelerometer. (b) Time and time-frequency responses of the cantilever sensor. (c) PSD functions.
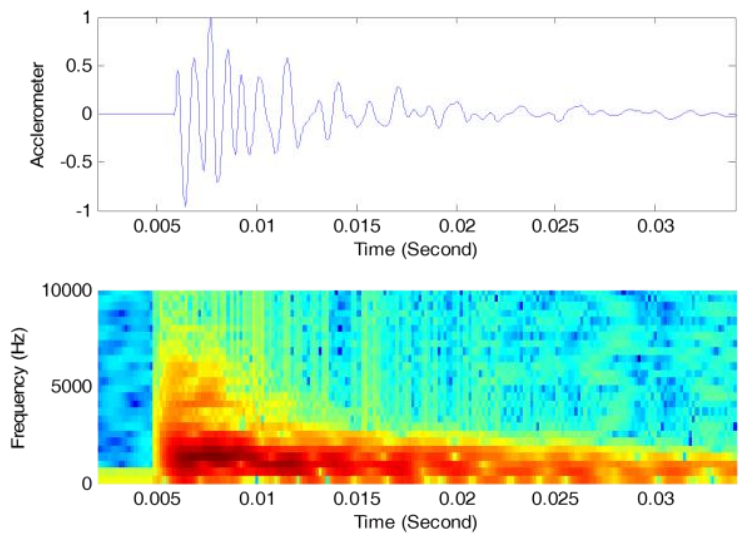

(a)
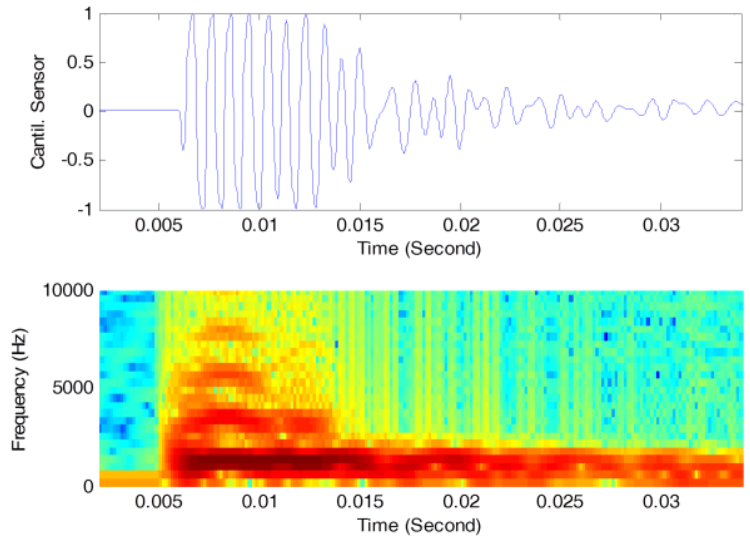

(b)

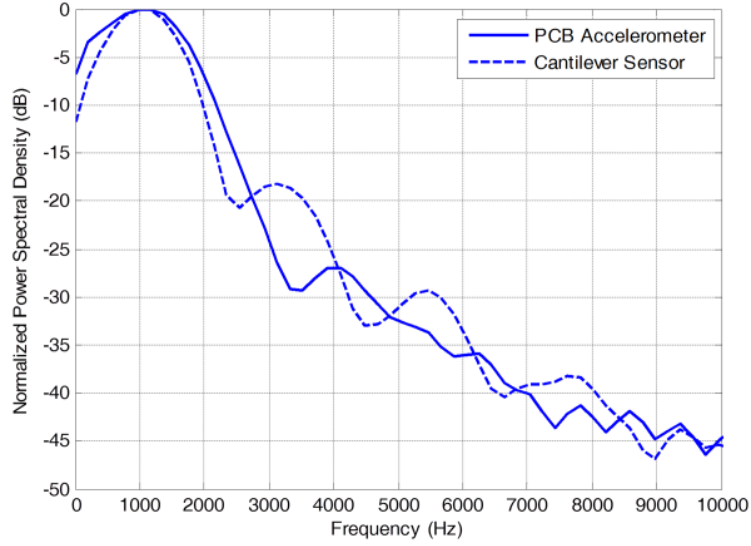

(c)

Fig. 10. Measured weak-impact responses of the two sensors on the $M D F$ plate. (a) Time and time-frequency responses of the accelerometer. (b) Time and time-frequency responses of the cantilever sensor. (c) PSD functions.

one measured at the glass plate as it can be seen from Fig. 9c, but the PSD of the cantilever sensor response at the MDF plate is quite similar to the one at the glass plate.

From Fig. 10c, the $Q$ factor at the resonance from the PSD of the cantilever sensor on the MDF plate is $Q=0.981$, which is smaller than that of the glass plate. Thus the reduced value of the $Q$ factor for the MDF plate represents that the MDF plate has 


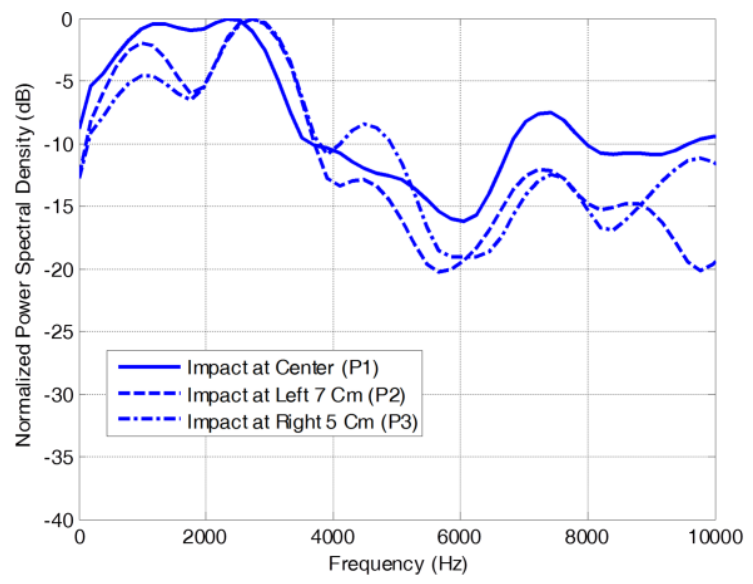

(a)

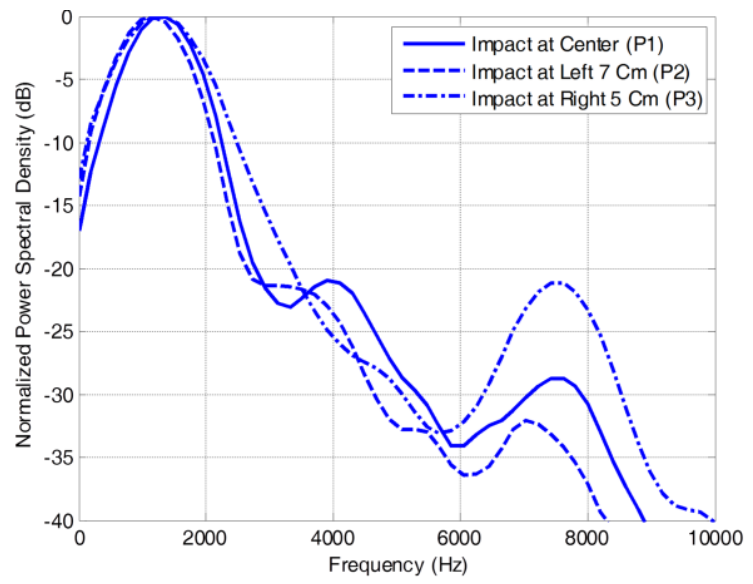

(b)

Fig. 11. Comparison of the PSDs of the measured weak-impact responses at 3 different locations $\mathrm{P} 1, \mathrm{P} 2$ and $\mathrm{P} 3$ on the glass plate. (a) Accelerometer. (b) Cantilever sensor.

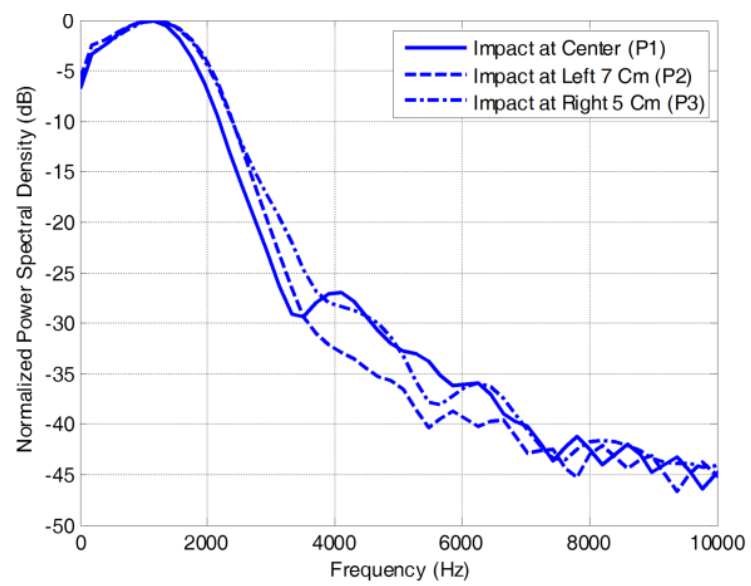

(a)

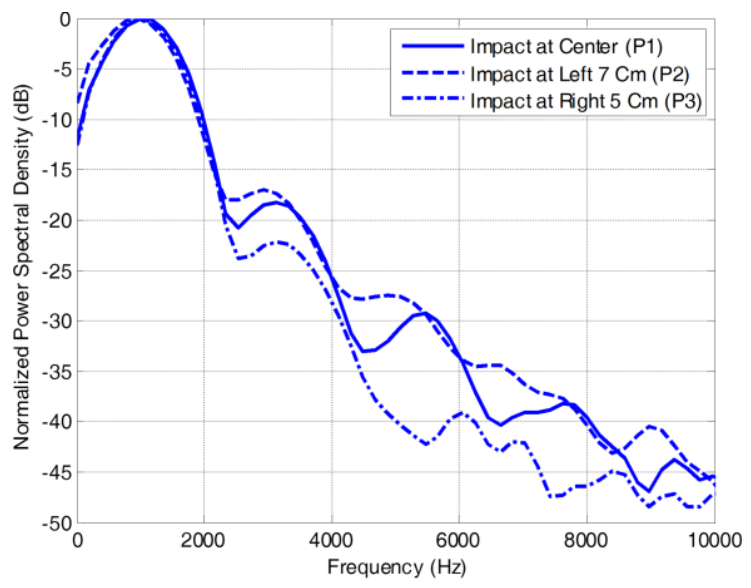

(b)

Fig. 12. Comparison of the PSDs of the measured weak-impact responses at 3 different locations $\mathrm{P} 1, \mathrm{P} 2$ and $\mathrm{P} 3$ on the $M D F$ plate. (a) Accelerometer. (b) Cantilever sensor.

more damping than the glass plate.

Hence, the weak-impact responses with the two sensors on the two different plates indicate that the cantilever sensor can provide much more constant outputs against the weak-impact than the accelerometer. The power spectral distribution of the cantilever sensor response is concentrated on a specific frequency range around $1,000 \mathrm{~Hz}$ whether the plate is an MDF or a glass, while that of the accelerometer response depends upon the plate dimension and material as can be seen from Figs 9c and 10c.

Figures 11 and 12 show the normalized PSD functions of measured responses of each sensor when the weakimpact locations vary as $\mathrm{P} 1$ (the center of the circle $R=15 \mathrm{~cm}), \mathrm{P} 2(7 \mathrm{~cm}$ left from $\mathrm{P} 1)$ and $\mathrm{P} 3(5 \mathrm{~cm}$ right from $\mathrm{P} 1)$, which are defined in Fig. 8. From these 3 different weak-impact locations, it is verified from Figs $11 \mathrm{~b}$ and $12 \mathrm{~b}$ that the cantilever sensor can provide a constant narrow-band response around $1,300 \mathrm{~Hz}$ at the glass plate or $1,000 \mathrm{~Hz}$ at the MDF plate whether the location is P1, P2 or P3. By the way, the accelerometer gives completely different frequency responses when the plate dimension and material vary as shown in Figs 11a and 12a. Thus the newly developed cantilever type sensor is more relevant to offer the narrow-band property for locating a weak-impact point using the TDOA approach. So it is proved experimentally that the cantilever type sensor responds consistently only to a specific narrow-band base vibration regardless of impact locations and the dimension and material of a plate. 
From the above experiment results, since the flexural wave velocity of a plate due to a weak-impact on a plate is dispersive, the mechanical narrow band-pass filtering of the cantilever sensor against the weak-impact on the plate allows the wave velocity constant. Thus in terms of the wave velocity, the cantilever sensor provides much better and more constant responses than the accelerometer.

In addition, this could be interpreted, especially for the rigid thin plates such as a glass or steel, that the cantilever sensor maybe more suitable to implement a large area touch panel. This is because the cantilever sensor is sensitive at detecting a lower frequency response as a long wavelength flexural wave can be propagated even longer on plates with less damping. Furthermore the design concept of the cantilever type piezoelectric sensor can allow designing the variation of the narrow-band by adjusting the fundamental natural frequencies of the cantilever beam.

\section{Conclusions}

A newly developed piezoelectric cantilever sensor to locate a weak-impact point on the two different plates of a glass and an MDF was presented, which is suitable for implementing a touch panel with the TDOA approach. Since this approach requires a constant flexural wave velocity for precise locating on the plate, the sensor was designed as a cantilever type to provide a narrow-band in order to overcome the dispersion property of the flexural vibration wave on the plate. Then the sensor was manufactured with a steel beam and a single crystal PMN-PT transducer. Then the sensor was attached on the plates and tested together with a commercial accelerometer.

The test results indicate that the cantilever sensor can provide a mechanical band-pass filtering regardless of the dimension and the material of the plate. It was proved that the cantilever sensor responses on the both plates showed a tonal component was strongly involved when the weak-impacts were imposed on the plates. However the accelerometer responses, which were also attached on the plates, gave a narrow-band property only on the MDF plate but showed a broadband property on the glass.

Thus, this could be interpreted, especially for the rigid thin plates such as a glass or steel, that the cantilever sensor maybe more suitable to implement a large area touch panel. This is because the cantilever sensor is sensitive at detecting a lower frequency response as a long wavelength flexural wave can be propagated even longer on plates with less damping. Furthermore the design concept of the cantilever type piezoelectric sensor can allow designing the variation of the narrow-band by adjusting the fundamental natural frequencies of the cantilever beam.

\section{Acknowledgments}

This work was supported by Mid-career Research Program through the National Research Foundation of Korea (NRF) grant funded by the Korean government (MEST) (No. 2009-0084728).

\section{References}

[1] A.K. Bhowmik, Z. Li and P.J. Bos, Mobile Displays: Technology and Applications, Wiley, 2008.

[2] C.K. Lee, Theory of laminated piezoelectric plates for the design of distributed sensors/actuators. Part I: Governing equations and reciprocal relationships, Journal of Acoustical Society of America 87(3) (1990), 1144-1158.

[3] C.K. Lee and T.C. O'Sullivan, Piezoelectric strain rate gages, Journal of Acoustical Society of America 90(2) (1991), 945-953.

[4] C.K. Lee, W.W. Chiang and T.C. O’Sullivan, Piezoelectric modal sensor/actuator pairs for critical active damping vibration control, Journal of Acoustical Society of America 90(1) (1991), 374-384.

[5] J. Rex and S.J. Elliott, The QWSIS-A new sensor for structural radiation control, Proceedings of MOVIC, 1992, pp. $339-343$.

[6] Y.-S. Lee, Active Control of Smart Structures using Distributed Piezoelectric Transducers, PhD Thesis, University of Southampton, 2000 .

[7] Q.-M. Wang, X. Du, B. Xu and L.E. Cross, Theoretical analysis of the sensor effect of cantilever piezoelectric benders, Journal of Applied Physics 85(3) (1979), 1702-1712.

[8] D. Isarakorn, M. Linder1, D. Briand and N.F. de Rooij, Evaluation of static measurement in piezoelectric cantilever sensors using a charge integration technique for chemical and biological detection, Measurement Science and Technology 21(7) (2010), 075801.

[9] M. Liu, J. Tong, L. Wang and T. Cui, Theoretical analysis of the sensing and actuating effects of piezoelectric multimorph cantilevers, Microsystem Technologies 12(4) (2006), 335-342. 
[10] J.S. Wilson, Sensor Technology Handbook, Newnes, 2005.

[11] W.T. Thomson and M.D. Dahleh, Theory of Vibration with Applications, (5th ed.), Prentice-Hall, 1997.

[12] Piezo Film Sensors Technical Manual, Measurement Specialties Inc., 2008.

[13] C.H. Knapp and G.C. Carter, The generalized correlation method for the estimation of time-delay, IEEE Trans Acoust, Speech, Signal Processing 24 (1976), 320-327.

[14] A.O. Hero and S.C. Schwartz, A new generalized cross correlator, IEEE Trans Acoust, Speech, Signal Processing 33 (1985), 38 -45.

[15] F. Fahy, Sound and Structural Vibration, Academic Press, 1985.

[16] J.F. Doyle, Wave Propagation in Structures, Springer, 1997.

[17] C.K. Lee, On Theory of Laminated Piezoelectric Plates for the Design of Distributed Sensors/Actuators, Journal of Acoustical Society of America 87(3) (1990), 1144-1158.

[18] R. Blevins, Formulas for Natural Frequency and Mode Shape, Krieger Publishing Company, 2001. 

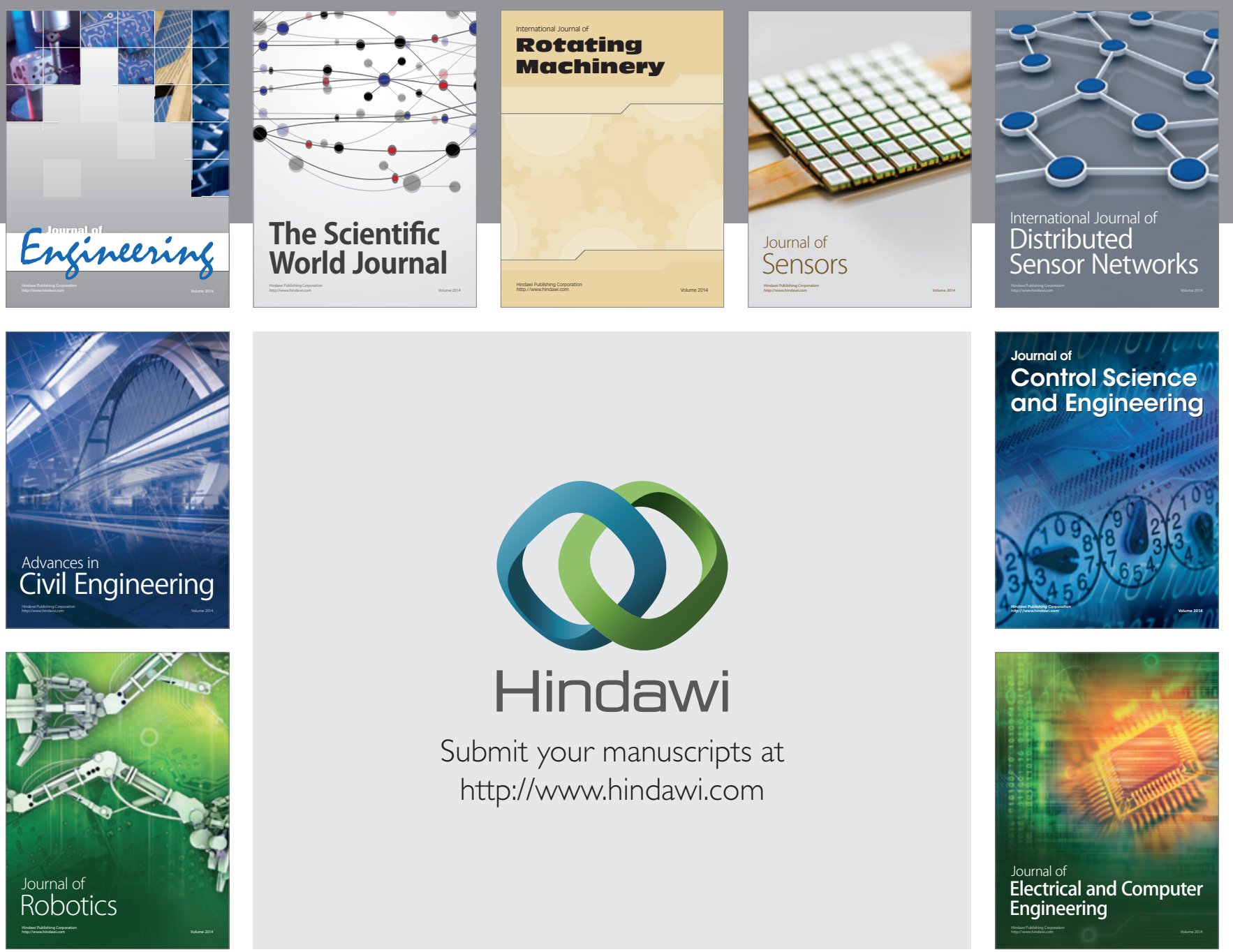

Submit your manuscripts at

http://www.hindawi.com
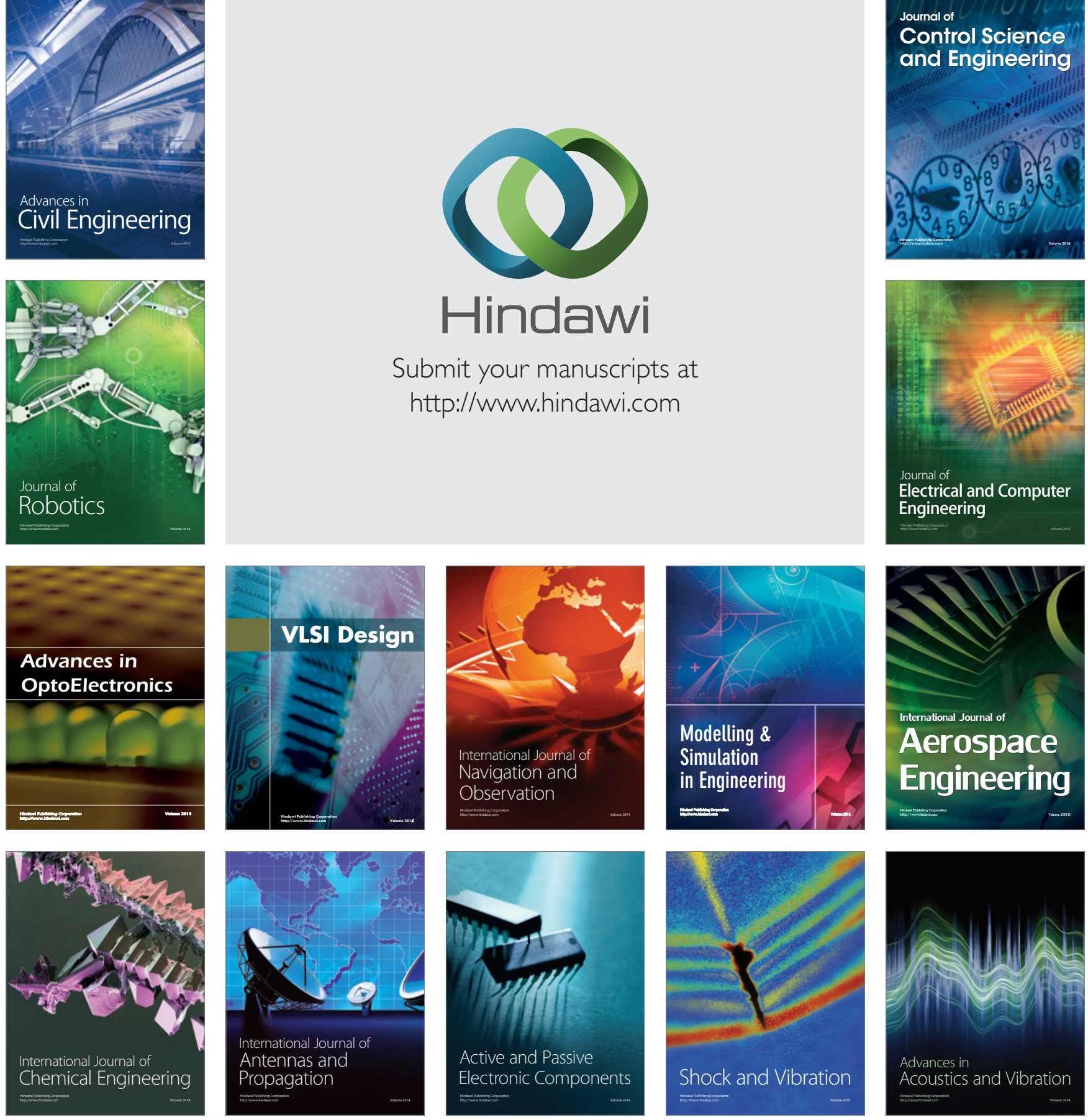